\title{
Les Trois Jours de la queue du dragon de Jacques Rebotier, entre théâtre, musique et poésie
}

Les Trois Jours de la queue du dragon ("The Three Days of the Dragon's

Tail") from Jacques Rebotier, over Boundaries between Theater, Music

and Poetry

\section{Nathalie Rannou}

\section{OpenEdition}

\section{Journals}

Édition électronique

URL : https://journals.openedition.org/recherchestravaux/793

DOI : $10.4000 /$ recherchestravaux.793

ISSN : 1969-6434

Éditeur

UGA Éditions/Université Grenoble Alpes

Édition imprimée

Date de publication : 1 décembre 2015

Pagination : 137-146

ISBN : $978-2-84310-314-8$

ISSN : 0151-1874

Référence électronique

Nathalie Rannou, « Les Trois Jours de la queue du dragon de Jacques Rebotier, entre théâtre, musique et poésie », Recherches \& Travaux [En ligne], 87 | 2015, mis en ligne le 01 janvier 2017, consulté le 29 octobre 2021. URL : http://journals.openedition.org/recherchestravaux/793 ; DOI : https://doi.org/ 10.4000/recherchestravaux.793 
Nathalie RANNOU

Univ. Grenoble Alpes

UR LITT\&ARTS

\section{Les Trois Jours de la queue du dragon de Jacques Rebotier, entre théâtre, musique et poésie}

À la question enfantine «Faut-il croire ce que l'on croit?", la parution du texte de Jacques Rebotier, Les Trois Jours de la queue du dragon, sous-titré "théâtre» semble entonner joyeusement l'air du soupçon. La prise en charge de l'illustration du volume en 2000 par sa fidèle collaboratrice Virginie Rochetti achève de brouiller les pistes qui permettraient d'affilier l'œuvre à la catégorie d'album-jeunesse, de poésie, de partition musicale, de cahier de jeux... Résolument, Jacques Rebotier nous offre, avec cette œuvre, le plaisir de déjouer les classifications éditoriales, littéraires, linguistiques mais aussi mentales et artistiques. Ce faisant, le poète-musicien-dramaturge invite le lecteur à une aventure régénérante : certes, on peut qualifier cette "hybridation générique ${ }^{\mathrm{I}}$ » de déconstruction, fidèle en cela à une veine majeure de la création artistique et théâtrale contemporaine, mais on peut aussi l'entendre comme une multiproposition offerte au lecteur, et examiner vers quels pactes de lecture combinés nous engage Jacques Rebotier en publiant cette œuvre chez Actes Sud-Papiers dans la collection «Heyoka jeunesse». Au-delà des questions du genre et de la réception qu'un livre aussi atypique réactive, c’est la dynamique générative de l'écriture que nous interrogerons dans un troisième temps.

I. L'expression «hybridation générique» est employée notamment par M. Bernanoce et A. Brillant-Annequin dans leur introduction au volume des actes du colloque Enseigner le théâtre contemporain, SCÉRÉN-CRDP de Grenoble, 2009, p. Io. 


\section{Une «hybridation générique» : la grande fête des "airs de famille»}

La recherche en théorie des genres ${ }^{2}$ ne cesse de se renouveler depuis les années 1990 où la fascination pour la postmodernité a pu faire croire à leur imminente disparition ${ }^{3}$. Le volume publié et la richesse d'invention en théâtre jeunesse nous rappellent cependant que le genre en soi n'est pas mort, mais qu'aucun n'obéit plus à des catégories formelles aux lignes préétablies. Cela fut souligné notamment lors du colloque de $2006^{4}$. Les spécialistes du genre littéraire tel Jean-Marie Schaeffer ${ }^{5} s^{\prime}$ attachent désormais à reconnaître des traits génériques, à la stabilité toute relative; la notion d' "airs de famille» reprise de Wittgenstein ${ }^{6}$ permet dès lors de dépasser les conceptions formalistes du genre. Marielle Macé rapatrie même la notion du côté de la réception en concluant tout naturellement que le genre, c'est ce que l'on en fait ${ }^{7}$. Jacques Rebotier n'est d'ailleurs pas le seul «inclassable». Dans sa notice consacrée à Trois Jours de la queue du dragon, Marie Bernanoce signale qu'Enzo Cormann et Pascal Rambert sont, comme Jacques Rebotier, musiciens tout autant qu'écrivains ${ }^{8}$. Mais ce dernier va au-delà du brouillage des genres, il en fait un sujet en soi. D’ailleurs, les premiers mots de la préface campent la problématique du genre comme un jeu :

Une pièce de théâtre? Une conférence? Ratée? Une circonférence? Du cirque? S'agit-il de dragons, qui s'agitent? Sans raison? De clarinettes? Avec déraison?

Est-ce une leçon de choses? De ténèbres? Est-ce la même chose? Est-ce du pas pareil $^{9}$ ?

En jonglant avec les apparences génériques de son texte, Jacques Rebotier développe un double mouvement : celui de l'hybridation foisonnante des «airs de famille» et celui de la responsabilisation du lecteur à qui s'offrent quantité

2. D. Coste, «Pourquoi les genres?» [en ligne]. Disponible sur Fabula, <http://www.fabula. org/revue/cr/42I.php> [consulté le 29/06/2007].

3. P. Ouellet, "Vie et mort du genre. Autopsie d'une survivance», dans La mort des genres, Montréal, La Nouvelle Barre du Jour, 1987.

4. M. Bernanoce, A. Brillant-Annequin, Enseigner le théatre contemporain, ouvr. cité.

5. J.-M. Schaeffer, Qu'est-ce qu'un genre littéraire?, Paris, Seuil, coll. "Poétique», I989.

6. L. Wittgenstein, Tractatus logico-philosophicus [192I], trad. P. Klossowski, Paris, Gallimard, coll. «Tel», I96I.

7. M. Macé, "Connaître et reconnaître un genre littéraire », Atelier de théorie littéraire, [en ligne]. Disponible sur Fabula [mise à jour le 24 mai 2007] <http://www.fabula.org/atelier> [consulté le 26/05/2008].

8. Ibid., p. 423.

9. J. Rebotier, Les Trois Jours de la queue du dragon, illustrations de V. Rochetti, Actes SudPapiers, coll. «Heyoka jeunesse», 2000, p. 5. 
de pactes de lecture possibles, en vertu d'une stylistique dont la particularité réside sans doute dans l'indécidable. Le lecteur chargé de l'appréciation générique du texte endosse alors une responsabilité, celle de décider du genre de ce qu'il reconfigure par sa lecture, en fonction des traits génériques qu'il aura privilégiés et combinés.

Nous avons donc entre les mains un livre de théâtre, ou du moins un texte qui en exhibe de nombreux signes. En effet, le contrat éditorial indique la nature fondamentalement théâtrale de l'œuvre : la collection «Heyoka jeunesse» rassemble, dit l'éditeur au seuil de l'ouvrage, "un répertoire théâtral ambitieux" capable de "conforter les passerelles nécessaires entre l'éphémère de la représentation et la mémoire de l'écrit». On apprend également, parmi les détails paratextuels, que la première représentation des Trois Jours de la queue du dragon aurait eu lieu en 1993, coproduite en 200I au Théâtre du Prisme, par le Centre dramatique national de Sartrouville et le Théâtre du Finistère à Quimper. Le nom du metteur en scène, Joël Jouanneau, mais également ceux des responsables lumière, son et décor sont avancés. Le terme de "rôle» qualifie la participation de Christian Drillaud à la création de l'œuvre. La prise en compte de l'usage scénique du texte ancre donc Les Trois Jours de la queue du dragon de plain-pied dans le théâtre joué. Dès lors, la répartition en trois parties rappelle une structure en actes ou en tableaux, un musicien quant à lui parlerait de «mouvements». Le recours à des sous-titres comme autant de scènes, la première phrase descriptive et narrative à vocation topographique ${ }^{\text {Io }}$, des énoncés infinitifs écrits en italiques comme "Monter-démonter les clarinettes» ou "Courir vite après sa queue, tous", donnent inévitablement un effet didascalique. L'énoncé "je regarde fixement» suivi de "Ils le font» en italiques, joue avec la notion ancienne de "didascalie interne». Certes, comme le souligne Anick Brillant-Annequin, «la distinction traditionnelle entre les genres dramatique et épique, établie par Platon et Aristote, auxquels s'est adjoint le lyrique et, à l'intérieur du dramatique, entre tragique et comique, est totalement brouillée ${ }^{\mathrm{II}} »$. Mais Jacques Rebotier va plus loin, il fait de cette démarche un moteur de création, il sollicite un lecteur actif et sensible au jeu du genre.

Il s'agirait donc de théâtre, mais dans une version ludique, décalée, pleine de jonglerie. Jacques Rebotier, à n'en pas douter, s’offre une liberté de clown, multiplie les grands écarts et l'équilibrisme stylistique. Il fait tournoyer son

IO. «Marcel Dragon se promène dans les rues de Tarascon. Il a des dents de devant. Il danse à un temps.» (Ibid., p. 7.)

II. A. Brillant-Annequin, «Le tragique, entre permanence et métamorphoses», ouvr. cité, p. 72. 
dragon, animal étrangement apprivoisé, à l'appellation inconstante ${ }^{\mathrm{i} 2}$. Il se comporte, c'est tentant, en parfait meneur de cirque, ou encore en cirqueconférencier. D'ailleurs, conformément à l'univers du cirque, la musique tient une place importante : l'instrument choisi, la clarinette n'est-il pas de ceux dont le clown blanc régala nos enfances? On note que les clarinettes aussi sont soumises au carnaval, c'est-à-dire à cette joyeuse déconstruction : tantôt démontées, elles sont cassées dix pages plus loin pour que l'on y joue deux morceaux à la fois, puis finalement, elles se retrouvent sans pavillon ${ }^{13}$. La danse qui ouvre le premier jour est dite, de façon cocasse «à un temps».

Compositeur, Rebotier ne laisse donc pas la dimension musicale au hasard de l'illustration du livre et de la représentation sur scène. Lors de la création de la pièce, c'est le quatuor Edison qui s'est prêté au jeu de cette «leçon de ténèbres», décidément baroque. Ce qualificatif implicite ne serait-il pas d'ailleurs une clé stylistique de l'œuvre : un baroque d'aujourd'hui qui défie, de sa liberté créative, le classicisme académique du langage maîtrisé et une part figée de la création contemporaine? On retient d'abord la précision de l'auteur : selon le projet énoncé en ouverture, les parties musicales correspondent à des codas, des fins de morceaux, comme autant de queues terminales. Or, le final, c'est le moment fort et flamboyant, l'instant de contact ultime entre l'œuvre et l'auditeur-spectateur, celui où se nouent et se dénouent le jeu et l'émotion de la réception. Multiplier les codas, dans un univers de dragons, c'est jouer avec sa queue, c'est indiquer paradoxalement que les bonnes choses n'ont pas une fin mais plusieurs, et que l'on n'en finit pas.

D'autre part, les partitions musicales intégrées dans le livre ne sont pas de simples jeux graphiques. Les deux notes fa-ré en clé d'ut reproduites en orangé servent à ponctuer l'œuvre comme autant de virgules musicales. Il s'agit les seize fois des mêmes notes, mais suivies d'un sous-titre qui varie. Seul «Le pil des pustules» semble résister au champ lexical de la musique ou du temps, tous les autres titres se jouant de la polysémie du mot "air », comme "Air entendu " ou "Air de rien $n^{\circ} 2$ ». On peut aussi observer la partition page 24. Elle repose sur une logique de répétition-variation : les trois phrases suivent la même mélodie avec un demi-ton de décalage croissant. Ce qui amuse le lecteur-musicien est la conjonction entre les paroles «trois p'tits points?! de la troisième phrase et le silence musical qui l'accompagne, comme si les instruments avaient déjà fait leur part en jouant jusqu'au bout les deux premières lignes. Plus loin, la portée est également triple, on peut donc imaginer la partie jouée par trois

I2. «Marcel Dragon», p. 7; «dragon Wilfried» et «Dragon le Marcel», p. 9; «Raoul R. Dragon», p. Io.

I3. Les clarinettes sont "démontées", p. I2; "cassées", p. 25; «sans pavillon », p. 30. 
clarinettes. Outre le dessin des petits dragons qui vient trahir le sérieux de la page, on remarque l'écriture de trois "flzg", c'est-à-dire, dans le vocabulaire de la musique contemporaine, trois Flatterzunge ${ }^{\text {T4 }}$ dont le jeu d'un musicien consciencieux imite, sans le savoir, le cri râpeux du dragon. Ainsi le morceau s'achève sur la «Bénédiction. Maudit sois-tu, Dragon le rogneux!». Or, si d'après l'exposition de l'œuvre, il faut lire ces partitions comme des cantiques de procession, il est impossible là encore de nier l'esprit carnavalesque, dans le sens bakhtinien du terme.

Par ailleurs, une série de traits génériques relie Les Trois Jours de la queue du dragon, de façon tout aussi ludique bien sûr, à la leçon scolaire. Le travail de l'illustratrice y contribue en figurant des frises explicatives, des schémas, des images mathématiques de numération ${ }^{15}$ ou encore un tableau de conjugaisons taché d'encre. L'expression «leçon de choses» est employée dans la préface, et le dragon y est effectivement présenté dans sa catégorie animalière - «Le dragon est un serpent»-, flanqué du traditionnel attribut du sujet. Cependant, la définition analogique et biologique tourne très vite à la fantaisie voire à l'ironie : «Un dragon est un serpent qui a une gueule.» La leçon de choses ne serait-elle qu'une supercherie pour faire admettre aux enfants toute la féerie de la nature que leur bon sens soupçonnerait d'inanité? Les consignes comme "Apprenez par cœur pour lundi le verbe être», ou les fiches de révision en vue des «Rogations» reprennent et détournent les stéréotypes stylistiques des genres scolaires. Les pages d'exercices n'ancrent pas davantage la «leçon» du côté du sérieux et prennent place dans l'œuvre comme modèles génériques bons à retourner : par exemple, on se demande qui, à part Ionesco, aurait demandé à ses personnages d'apprendre "par cœur la phrase suivante : quand les souris chantent, les souris chantent» dans $l^{\prime}$ «Exercice ${ }^{\circ} 3$ ". Il est clair que Jacques Rebotier nous livre ici la magistrale introduction à son "Cours complet de bêtise», mais contrairement à ses aînés, l'auteur fait du sentiment de l'absurde, devant notre condition et notre langage, un programme ludique, un exutoire heureux qui transcende totalement le problème de l'appartenance générique du texte.

14. En écriture de la musique contemporaine pour instruments à vent, le Flatterzunge réclame un coup de langue particulier ou un roulement de gorge qui dénature le timbre habituel afin de produire un son granuleux. O. Messiaen par exemple en fait usage pour évoquer certains cris d'oiseaux.

I5. Ce type d'image se trouve p. II, 20, 36. 


\section{Des pactes de lecture ouverts : à l'écoute de la poésie du texte}

La question du brouillage des genres prend un intérêt démultiplié si on y adjoint une réflexion sur la lecture. De fait, depuis les travaux incontournables de Michel Picard ${ }^{16}$, les types d'engagement du lecteur sont comparables à différentes façons d'appréhender le jeu. À ce titre, on peut donner aux dimensions ludiques du texte de Jacques Rebotier une portée métaphorique quant à la lecture elle-même. Le jeu n'est pas seulement celui de l'acteur-circonférencier ou des joueurs de clarinette. Il est aussi présent à travers les mots fléchés, la maquette de dragon à découper, le labyrinthe, ou encore la devinette sous forme de texte à trou : "C'est en draguant que l'on devient... ${ }^{17}$ » Le jeu est dans la vie, dans l'enfance, omniprésent et stimulant, occasionnant l'adhésion ou l'écart du second degré; et c'est en jouant que l'on fait littérature, quelle que soit la nature de l'objet créé.

Toujours en référence enjouée aux théories littéraires actuelles, on relève à quel point le texte est ainsi concrètement "troué». Si, dans l'esprit des sémiologues et des phénoménologues de la lecture ${ }^{18}$ le travail du récepteur consiste à inférer en comblant les blancs du texte, voici avec Les Trois Jours de la queue du dragon une mise à l'épreuve au pied de la lettre. Les fins de section sur les termes "À suivre», "et cætera», "trois petits points», "(illisible) », confient au récepteur la mission de l'achèvement. La discontinuité toute baroque du texte, en lien avec une esthétique du fragment et de l'hétérogène pointée par David Marron $^{19}$ implique également cette participation du lecteur à qui il revient de décider du fil directeur, des liens logiques et du propos effectif de l'œuvre.

En réalité, la façon sans doute la plus englobante et ouverte de recevoir ce livre est de le lire comme on aborde un poème contemporain. Poétique, le texte l'est déjà par sa mise en page qui joue des marges, des retours à la ligne et d'une organisation de l'espace du livre par un système alliant blocs de texte, telles des strophes de vers libres, et repos sur le blanc. La dimension visuelle de l'écriture poétique se manifeste à de nombreuses reprises dans d'autres œuvres de Jacques Rebotier. On peut être sensible par exemple à la mise en

I6. M. Picard, La lecture comme jeu, essai sur la littérature, Paris, Minuit, coll. "Critique», 1986.

I7. J. Rebotier, ouvr. cité, p. 20.

I8. Que l'on songe à U. Eco notamment ou à W. Iser pour qui le texte programme l'intervention participative du lecteur dans les failles stratégiques du texte.

19. D. Marron, «Les 47 autobiographies de Jacques Rebotier ou comment se raconter poétiquement et jouer de la déroute», Recherches \& Travaux, n ${ }^{\circ} 75,2009$, p. I23-I33. 
page de «I2 essais d'insolitude», dans Le Moment que ${ }^{20}$. Cette section est écrite matériellement de façon flottante sur des lignes onduleuses invisibles, telles des vagues (à l'âme), comme si l'intonation du lecteur était invitée à monter et à descendre au gré d'une portée. Les Trois Jours de la queue du dragon aussi engage volontiers l'oralisation du texte : «Prononcez à voix haute :- un ragon - une larinette ${ }^{21} »$. Dès lors, la dimension visuelle rejoint la dimension musicale et sonore.

De fait, l'aspect poétique tient à la permanence des effets de sonorités : dès la page 5 , l'assonance en [ô] draine l'écriture en lui imprimant un air de ritournelle ou de comptine. Et si l'on songe à la définition jakobsonienne de la fonction poétique du langage, on mesure à quel point elle constitue un levier principal de la lecture. Le plaisir des sons et de la formule décroche le langage de son utilitarisme et de toute référentialité autre que celle au langage lui-même : «Un dragon très gras drague un gros dragon gradé, c'est dégradant ${ }^{22}$.» On mesure à l'extrême ce plaisir du matériau verbal et visuel jusqu'à l'imprononçable et l'illisible dans le dessin des "bruits que fait le dragon ${ }^{23}$ ».

En cas de réception poétique, le besoin de narration, le mimétisme et la fonction référentielle du langage sont fortement atténués. Chez Jacques Rebotier, quand le propos ressemble au discours sérieux de la philosophie, ce n'est pas pour référer mais pour produire des syllogismes poétiques rythmés, tronqués et fantaisistes :

Un circonflexe est l'inverse d'une hirondelle.

Une hirondelle est l'inverse d'un dragon.

Un dragon est un circonflexe ${ }^{24}$.

On peut noter que la philosophie ou plus exactement la logique n'est pas la seule science dont le sérieux vole en éclat : la psychanalyse et les pulsions du «ça» sont pris tout autant dans le filet carnavalesque. C'est donc la fonction poétique qui l'emporte ainsi que le plaisir du détournement et du jeu avec la matière sonore.

Cette façon de concevoir la poésie comme éveil plaisant à la matérialité du langage et des mots encourage l'invention verbale. Les néologismes sont légion : "ragons-larinettes", "fêtise», "têtise», par exemple. Ils contribuent à la dimension imaginaire de l'œuvre et à sa fantaisie, tout en l'ancrant parfois dans une intertextualité ludique : «l'ancien rituel des Rogations» renvoie à la

20. J. Rebotier, Le Moment que, Marseille, cipM/Spectres Familiers, coll. «Le Refuge», 1998.

2I. Ibid., p. 23.

22. Ibid., p. I4.

23. Ibid., p. I6.

24. Ibid., p. 24. 
Légende dorée 25 " "Le dragonique» qui "a dragonné tout l'étée ${ }^{26}$ » ressemble à s'y méprendre à une certaine cigale. Dans ce bestiaire particulier, l'ombre de La Fontaine n'est jamais loin, l'inventaire à la Prévert non plus :

Un dragon a un bec, un barillet, un pavillon, plusieurs corps, des anneaux, plein de pattes, et des tampons.

Et un écouvillon ${ }^{27}$.

D'ailleurs un «écouvillon» n'est-il pas une sorte de raton laveur pour clarinette?

Enfin, à la croisée des réceptions poétique et théâtrale de l'œuvre se tient l'expérience de la parole performative. Car non seulement Jacques Rebotier participe volontiers du courant de la poésie sonore en réalisant des performances artistiques, mais son écriture elle-même met à l'épreuve la performativité du langage. Le lecteur accomplit, par l'énonciation que produisent sa lecture et son imaginaire, une parole en acte, une énonciation performative : «je regarde fixement. D'un œil tout à fait perçant. Ils le font ${ }^{28}$ ». La singularité qui s'exprime alors est l'espace-temps de la réalisation poétique : dans une réception du texte reçu en tant que poème, elle se fonde dans l'ouverture du désir et la sensibilité du lecteur lui-même à l'expérience d'une parole active.

\section{Une dynamique générant de la parole}

En définitive, qu'il soit lu intérieurement comme un poème ou imaginé, énoncé et joué sur une scène, le texte tient par sa "fantaisie jubilatoire», selon la qualification que Corinne Frassetti accorde à La Tortue géante des Galapagos de Rébecca Dautremer, et par la dynamique d'une production langagière très prolixe. Cette génération de la parole puise dans des ressources stylistiques parfois multiséculaires, héritière des grands rhétoriqueurs $\mathrm{du} \mathrm{Xv}^{\mathrm{e}}$ siècle qui savaient ciseler la langue sur toutes ses faces ou, tout proches de nous, des Oulipiens. Si l'on entreprenait de mettre au clair la poétique de Jacques Rebotier, il faudrait identifier la variété des figures de sens, de construction et de sonorités qui génèrent cette écriture complexe qui ne semble pourtant jamais se prendre au sérieux.

25. Les Rogations constituent une pratique processionnaire préchrétienne. J. de Voragine décrit les Rogations dans le chapitre «La Litanie majeure et la litanie mineure» : "on y porte la croix, on sonne les cloches, on porte la bannière; en quelques églises on porte un dragon avec une queue énorme» (La Légende dorée, t. I, trad. J.-B. Roze, Paris, Garnier-Flammarion, I967, p. 353).

26. J. Rebotier, ouvr. cité, p. 22.

27. Ibid., p. I2.

28. Ibid., p. Iо. 
Le texte doit d'abord beaucoup au potentiel polysémique de la langue, à l'écart entre les sens premiers, matériels, concrets et l'usage souvent dominant que l'on fait des mêmes mots ou expressions : «se passer les morceaux» de clarinette, dans ce "Cours complet de bêtise», nécessite de mettre l'instrument en pièces de façon à en faire circuler les bouts, de main en main. Les «guillemets» donnent lieu à des ouvertures et des fermetures musicales ${ }^{29}$. Le poète réveille les images usées, il réactive les concrétisations imageantes que l'habitude langagière avait éteintes. Les prépositions par exemple reprennent vie comme lorsqu'il s'agit de se promener dans les rues avec «un air de rien ». L'air de rien devient non plus une façon d'être mais une entité avec laquelle on chemine.

Le jeu de réactivation des mots usés s'effectue aussi à partir de paronymies : «suivez le vide», nous demande-t-on page I2. Page Io, le dragon se promène non pas à Boulogne mais au «bois de Boulons». L'effet humoristique tient au malin plaisir que trouve le lecteur à repérer le stéréotype-source que l'auteur a détourné. Rebotier nous livre d'ailleurs la clé stylistique de ses choix à travers le nom du "Jardin d'approximation" où l'on s'acclimate. L'esthétique fort travaillée de l'à-peu-près participe de l'insoumission générale aux formes, aux lieux ainsi qu'aux conventions littéraires, culturelles, langagières. Les motsvalises (parfois approximatifs) font partie de la fête : le "ragon-larinette» est un des néologismes clés de l'œuvre.

Le calembour, comme dans "S'agit-il de dragons qui śagitent» et l'anagramme font partie des moteurs créatifs, que les deux mots soient présents, - «Il lance des signes aux singes ${ }^{3 \circ}$ »-, ou que le terme source reste implicite mais fort reconnaissable grâce au caractère figé de la référence : «le Prince marchant». Les anagrammes amusent le lecteur qui reconnait les déplacements de lettres et de sons. Ils appartiennent à la famille des rimes acrobatiques, batelées, annexées, équivoquées, vers léonins et autres splendeurs poétiques dont on imagine Rebotier s'abreuver. D'ailleurs, le poète trouve des solutions pour approcher la rime emperière ${ }^{3 \mathrm{I}}$ : "bêtise, fêtise, têtise." Il lui suffit de combiner répétition, néologisme et approximation. D’innombrables passages de l'œuvre offrent des calembours couplés à des chiasmes : « lire entre les dires, dire entre les lignes ${ }^{32} »$.

\footnotetext{
29. Ibid., p. I3.

30. Ibid., p. Io.

31. "On dit que la rime est emperière quand la syllabe de rime est triplée». M. Aquien s'appuie sur un exemple de T. Sébillet. M. Aquien, Dictionnaire de poétique, Paris, Le Livre de Poche, 1993, p. 236. "bêtise, fêtise, têtise se trouve p. 22.

32. J. Rebotier, ouvr. cité, p. 37.
} 
Un autre moteur stylistique réside dans l'amplification. Elle peut jouer à l'échelle du mot - «cet en train-train ${ }^{33}$ »-, du groupe de mots, mais surtout au niveau du discours. Page 2I est répété par trois fois le début de paragraphe : «Le dragon s'efforce de symboliser les forces primitives... ». La seconde occurrence varie cependant : «puissances» remplace «forces». Il est tentant de reconnaître dans ce moteur d'écriture la logique de la musique répétitive et sérielle du $\mathrm{XX}^{\mathrm{e}}$ siècle. Cela dit, la reprise syntaxique est aussi un principe de la comptine, et l'on frissonne à l'idée de se promener "dans les bois" (même "de Boulons») lorsque l'on découvre que le dragon, comme le loup de la chanson enfantine, s'habille par étapes : «je mets mon Tarascon [...] je mets mon panty [...] je mets mes écailles ${ }^{34}$ ».

Sur le plan sémantique enfin, le texte s'alimente d'improbables croisements d'isotopies : entre musique et grammaire - "on en jouait lors des ouvertures de guillemets ${ }^{35} »-$, ou entre musique et mathématiques, plus particulièrement page 15 : «LA CLARINETTE EST LA RACINE CARRÉE DE LA CLARINE, ET LA RACINE CUBIQUE DU CLAIRON. ON PEUT AUSSI PARLER DE "PUISSANCE"." Cette jubilation linguistique alimente le foisonnement imaginaire en créant des croisements d'univers improbables et jaillissants.

On peut souligner pour terminer un procédé qualifiable de métanéologisme : en annonçant vouloir "écrire quelque chose d'entièrement caudiforme $e^{36}$ ", le poète applique immédiatement, de façon performative, son propre principe. Ce qui est fantastique, c'est que le lecteur, d'un même mouvement, comprend et acquiesce à ce néologisme comme on adhère au monde et au langage de l'évidence.

Le lecteur me permettra-t-il de terminer cet article sans véritable conclusion ni coda? Il faut dire qu'après avoir examiné la question des genres, la dimension poétique et quelques-uns des principes stylistiques moteurs de ce livre, nous arrivons au quatrième jour et la queue du dragon s'est sauvée au-delà des 25000 signes (ou singes) autorisés. J'ai peut-être aussi pris la leçon libertaire de Jacques Rebotier un peu trop... au sérieux... en dépit de l'élan carnavalesque que l'auteur offre de vivre avec entrain à des lecteurs de tous âges. "(Faire ici une action de disgrâce ${ }^{37}$ (Ils sourient).»

33. Ibid., p. 19 .

34. Ibid., p. II.

35. Ibid., p. I3.

36. Ibid., p. 5.

37. Ibid., p. 38 . 